\title{
Kernwachstum und Nukleolengröße bei den Eiern von Lima hians (Lamell.) Ein Beitrag zur Frage der partiellen Systeme Von
}

L. H. Bretschneider und Gottwalt Christian Hirsch

Aus der Abt. f. exper. Morphologie des Zool. Labor., Utrecht, Holland

Eingegangen ant 13. Oktoker $193^{6}$

Die R. HeRTwIG'sche Frage nach der „Kern-Plasma-Relation“ ist ein interessantes cytologisches Problem. Dasselbe gilt von der ,Kern-Nukleolus-Relation“"

Der Kern umschließt als höheres System das partielle System des Nukleolus, Kern und Nukleolus stehen damit in irgendeinem Verhältnis zu einander. Gleichzeitig steht der Nukleolus in irgendeinem Verhältnis zu den anderen Partialsystemen des Kernes: z.B. zu dem Linin, dem Kernsaft, dem Chromatin.

Zur Aufhellung dieses Verhältnisses des Nukleolus einerseits und anderseits der übrigen Partialsysteme und dem großen System Kern, können nun verschiedene Wege beschritten werden. Diese Wege kann man in physiologische und morphologische einteilen:

1. Physiologisch kann die Beziehung der Nukleolen zu den übrigen Partialsystemen der Zelle untersucht werden durch Anwendung chemischer und physikalischer Methoden. Es könnte dadurch in Zukunft einmal z.B. der Stoffaustausch zwischen Kern und Nukleolus deutlich gemacht werden.

2. Besteht die Möglichkeit, morphologische Wege zu beschreiten. Hier sehen wir vorallem zwei Fragen: es kann in erster Linie die Entwicklung der Nukleolen näher untersucht werden, d.h. die Frage, ob die Substanz der Nukleolen in Relation steht zu anderen partiellen Systemen des Kernes oder des Plasmas, so wie die Untersuchungen von H. BAUER und M. LENOIR uns darüber kürzlich aufgeklärt haben. Gleichzeitig könnte damit die Frage der Determination in Angriff genommen werden. Auch diese Fragen sollen uns hier zunächst noch nicht beschäftigen.

Dagegen wollen wir einen zweiten morphologischen Weg beschreiten. Wir haben uns gefragt, ob in dem Verhältnisse der Größen des Kerns und seines Nukleolus irgendeine Gesetzmäßigkeit zu finden ist. Diese Frage nach dem Größenverhältnisse erscheint uns eine ebenso interessante Frage wie die Kern-Plasma-Relation, 
denn sie ist enthalten in der allgemein-biologischen Frage nach den partiellen Systemen, welche die Zelle (und in den höheren Ordnungen auch den ganzen Organismus) aufbauen.

Was bedeutet nun diese Frage nach den partiellen Systemen? Der Begriff ist von Avenarius gebraucht worden um auszudrücken, daß die Welt aufgebaut ist aus Systemen verschiedener Größenordnung, welche so in einander geschachtelt sind, daß ein System höherer Ordnung mehrerer Systeme kleinerer Ordnung umfaßt und zu einem neuen Ganzen vereinigt, eine Tatsache, welche auch durch den Begriff der Enkapsis ausgedrückt wird. Um bei unserem kleinen Beispiel zu bleiben: es stellt zweifellos der Nukleolus irgendein „Partialsystem“ des höheren Systems Kern dar, denn der Kern umfaßt den Nukleolus. Ob dieses System noch als „lebend“ oder ,nicht-lebend“ (G. HeRTwIG) anzusehen ist, spielt hierbei zunächst keine Rolle, da die Meinung hierüber abhängig ist von der Frage, was man überhaupt als ,lebend“ betrachtet. Wohl aber ist die Frage erforschbar, in welchem Verhältnisse das partielle System Nukleolus zu dem höheren System Kern physiologisch und morphologisch steht. Eine Antwort auf diese Frage kann dadurch gegeben werden, daß man, wie oben auseinandergesetzt, das physiologische und morphologische Verhältnis dieser beiden Systeme näher untersucht. Und bei dieser Untersuchung spielt denn die Frage nach dem Größenverhältnisse dieser beiden Systeme in der Tat eine Rolle. Es gibt nämlich für ein solches höheres System (wie den Kern) und seine Partialsysteme (wie z.B. den Nukleolus) hinsichtlich ihrer gegenseitigen Größe zwei verschiedene Möglichkeiten:

1. Beide Systeme können in einem festen Größenverhältnisse zu einander stehen. Dann besitzt das kleinere partielle System keinerlei Autonomie hinsichtlich seiner Masse, sondern es ist ein in der Masse abhängiger kleinerer Teil des größeren Systems. Bei zunehmender Kerngröße müßte also auch der Nukleolus kleiner werden. Es würde sich also in diesem Falle um ein größeres System Kern handeln, welches größenbestimmend das Partial-System umgibt, wobei dies Partialsystem keine Massen-Autonomie besitzt, d.h. keine Möglichkeit, von sich aus seine Größe zu bestimmen; sondern seine Masse würde bestimmt werden allein von dem höheren System aus, von welchem es hinsichtlich seiner Masse abhängig ist.

2. Es besteht auch die Möglichkeit, daß das kleinere partielle System, der Nukleolus, hinsichtlich seiner Masse eine Autonomie besitzt gegenüber dem umfassenden höheren System, dem Kern; das heißt: das Schicksal des Nukleolus kann wohl in vieler Hinsicht von dem höheren System abhängig sein, also mancherlei Heteronomie zeigen.. ., aber wenigstens hinsichtlich der Größe, d.h. der Massen- 
zunahme und -abnahme, ist der Nukleolus als Partialsystem gegenüber dem höheren System Kern autonom.

Gewiß ist ein solcher Massenwechsel nur ein Kennzeichen unter vielen anderen Prozessen, die sich in einem Verhältnis wie zwischen Kern und Nukleolus abspielen können; aber wir sehen, daß die Frage: ist die Größe des Nukleolus abhängig von der Kerngröße? doch nicht ohne Bedeutung ist, und zwar als eine Teilfrage nach dem Verhältnisse der partiellen Systeme im Kerne.

Diese Frage nach der Abhängigkeit oder Unabhängigkeit der Nukleolengröße von der Kerngröße hat bisher auch schon einige andere Forscher beschäftigt und es haben sich hier zwei verschiedene Meinungen herausgebildet:

1. Vorallem M. HEIDENHAIN hat in seinem gedanklich so reich angelegten Buche „Plasma und Zelle“ die Meinung vertreten, daß die Nukleolenmasse abhängig wäre von der Kerngröße: ,die Beziehung der Menge der nuklearen Substanz zum Wachstum des Kernes, d.h. zur Vermehrung des Chromatins steht außer Frage". Hier ist also von der Vermehrung des Chromatins vorallem die Rede und sie ist gleich gesetzt dem Wachstum des Kernes. Wir glauben aber, daß man heute vorsichtiger sein muB und Chromatinvermehrung und Kernwachstum nicht mehr gleichsetzen kann. Es hängt die Größe des Kernes auch noch von mehreren anderen Faktoren ab, nicht nur von der Vermehrung des Chromatins. M. HEIDENHAIN führt mehrere Beispiele dafür an, wo sehr große Kerne auch entsprechend große oder zahlreiche Nukleolen besäßen, oder wo kleine Kerne auch nur kleine Nukleolen hätten. Und er schließt mit den Worten: „Unterliegt der Kern einem fortgesetzten Größenwachstum ohne in Mitose wiederum einzutreten, so muß die fortgesetzte P-speicherung im Sinne der angedeuteten Assimilationsprozesse auch $\mathrm{zu}$ einer fortgesetzten Neuerzeugung von Nukleolarsubstanz führen, was mit dem aus der Beobachtung ermittelten Verhältnisse der Menge der Nukleolarsubstanz zum Wachstum des Kernes völlig übereinstimmt" Auch M. LENOIR (1931) kommt bei Pflanzenzellen zu dem vorläufigen Schluß, daß sich die Partialsysteme Nukleolen, Chromatinnetz und Chromosomen in einem konstanten Gleichgewicht in der Zusammensetzung des Karyoplasmas befinden sollen.

2. Dem gegenüber haben eine Reihe von Autoren, vorallem JÖRGENSEN (1913) und O. HARTMANN (1921) zu beobachten gemeint, daß ein festes Größenverhältnis zwischen Kern und Nukleolus n i cht bestünde. M. JöRgensen kommt zu dem Ergebnis: ,die Zahl der Nukleolen und ihrer Masse ist unabhängig vom Wachstum des Kernes und von dex Intensität des Stoffwechsels zwischen Kern und Zelle“. Er führt als Argument für diese These aus seiner großen 
vergleichend -anatomischen Arbeit mehrere Fälle an, wovon das ,,schlagendste Beispiel für die Unabhängigkeit von Kernwachstum einerseits und Nukleolenwachstum andererseits" der Kern der Eier von Cobitis nach RHODE sein soll. Betrachtet man die Abbildung, so kommen einem Zweifel, ob denn in der Tat garkeine Beziehung besteht zwischen der Vermehrung der Kerngröße und der Nukleolenmasse; man zweifelt an der Durchschlagskraft dieses ,,schlagendsten Beispieles" Wir haben daraufhin das Verhältnis von Kerngröße und Nukleolenmasse in der von JöRGENSEN wiedergegebenen Zeichnung der Eizellkerne von Cobitis auf fotografischem Wege nachgeprüft, indem wir die Abbildung fotografierten, auf etwas stärkerem fotografischem Papier vergrößerten und einerseits die Eikerne, andererseits die Nukleolen ausschnitten. Dann wurde Nukleolenmasse und Kernmasse in Form des fotografischen Papieres gewogen. Es hat sich folgendes herausgestellt:

\begin{tabular}{l|lrrrr}
\hline Stadium & 1 & 2 & 3 & 4 & 5 \\
\hline Kern : & 8 & 10 & 28 & 32 & 77 \\
Nukleolen : & 0,7 & 3 & 7 & 17 & 40
\end{tabular}

Die Kurven dieser Zahlen laufen fast parallel; wenn man sie in der dritten Dimension berechnet so besteht hier wohl die Möglichkeit einer Abhängigkeit der Masse des Nukleolus von der Kernmasse!

Andere Eier dagegen zeigen schon rein nach optischer Schätzung in der Tat ein recht wechselndes Verhältnis der Nukleolus-Masse zur Kerngröße.

Auch O. Hartmann kommt 1921 an den Darm-, Ganglien- und Augenzellen von Sida zu dem Ergebnis, ,die Nukleolarkernrelation nimmt mit wachsender Zellgröße ab und zwar besonders stark bis zur Geburt; nach derselben findet nur mehr eine geringe Verkleinerung statt, während im höchsten Alter vielleicht ein sekundäres Anwachsen stattfindet. Das deutet darauf hin, daß bei Herabsetzung des zellulären Stoffwechsels ein Anwachsen der Nukleolen stattfindet; der Umstand, daß gerade z.Zt. der Geburt, also zu Beginn der Verdauungstätigkeit des Darmes, besonders stark Nukleolenreduktion stattfindet, spricht dementsprechend für eine Verkleinerung der Nukleolen bei intensivem Stoffwechsel (Verdauung)".

Wir glaubten daher dieser Frage noch einmal an Eiern nachgehen zu müssen mit einer etwas strengeren Methodik.

Wir haben nun ein Objekt gewählt, welches in den Eizellen stets nur einen einzigen Nukleolus besitzt; das sind die Eizellen der Lamellibranchiaten. Für eine genauere Technik ist es ferner notwendig, mit möglichst einfachen Mitteln die Masse zu berechnen. 
Das ist nur dann möglich, wenn sowohl die Zellkerne als auch die Nukleolen eine möglichst annähernde Kugelform besitzen; ist das nicht der Fall, so sind die Berechnungen natürlich viel komplizierter. Wir fanden in den Eiern von Lima hians ein geeignetes Objekt und legen im Folgenden unsere Ergebnisse vor zur Entscheidung der Frage, ob sich ein festes Größenverhältnis zwischen Kern und Nukleolus findet oder nicht.

\section{Technik}

Das Material stammt von der Feilmuschel Lima hians, welche in Juni 1933 auf der Zoologischen Station Rovigno durch den erstgenannten Autor in BouIN fixiert wurde, in Paraffin geschnitten 7,5 $\mu$, und gefärbt mit saurem Haemalaun-Eosin. Die Größenmessungen wurden sehr einfach vorgenommen, da es sich ja nur um eine Darstellung der relativen Größenverhältnisse handelt; von jedem Ei, dessen Kern in der Mitte seiner Kugel angeschnitten wurde und welcher gleichzeitig den Nukleolus in der enthaltenen Kugelhälfte zeigte, wurde mit einem einfachen Okularmikrometer der Durchmesser des Kernes und des Nukleolus bestimmt. Die beistehende Figur 5 gibt also auf der linken Seite die Anzahl der Teilstriche wieder.

\section{Ergebnisse der Beobachtung}

Abbildung 1-4 zeigt Plasma, Kerne und Nukleolen genannter Eier. Es ergibt sich, daß auch diese Eikerne in der längsten Zeit ihres Wachstums sogen. „Amphinukleolen“ besitzen (Abb. 3-4) über deren Schicksal wir später berichten werden. Abb. 1-4 zeigt ferner, daß die Größe des Kernes im wachsenden Ei regelmäßig zunimmt, wenigstens bis $\mathrm{zu}$ dem gewissen Endstadium, welches gemessen wurde; alte befruchtungsreife Eier sind noch nicht gemessen worden.

Die Größe der Nukleolen nimmt zu Anfang etwa in demselben Maße zu, wie die Kerngröße. Dann aber hat auf dem 5 . berechneten Größenstadium der Nukleolus seine vorläufig endgültige Größe erreicht, denn die Kurve steigt nicht mehr an, sondern bleibt auf der Höhe von etwa 5-6 Teilstrichen stehen, während der Kern gleichmäßig bis zu 30 Teilstrichen anwächst. Wir haben absichtlich davon abgesehen, die Größe räumlich $\mathrm{zu}$ berechnen.

Wir glauben nach dieser Beobachtung sagen zu können, daß das Kernwachstum und die Größe des Nukleolus bei diesem Objekt Lima hians unabhängig von einander sind: der Kern nimmt weiter stetig an Größe zu, während der Nukleolus alsbald auf einer vorläufig endgültigen Größe stehen bleibt. 


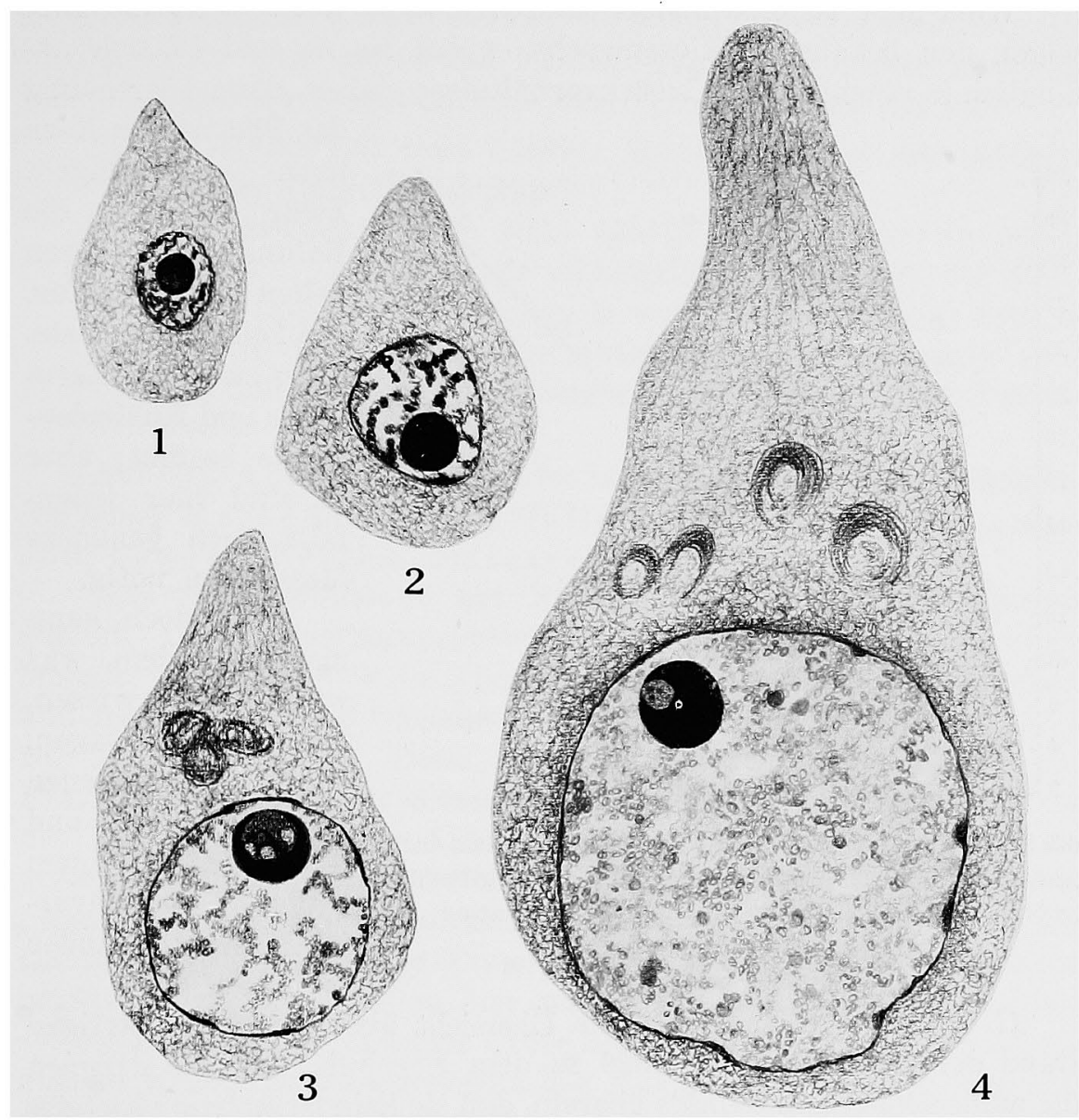

Abb. 1-4: Lima hians, 4 verschieden große Eizellen zur Demonstration des Verhältnisses zwischen Nukleolus, Kern und Plasma. 1: Eizelle, die sich eben zu differenzieren beginnt. 2: Erste Wachstumsperiode, in welcher das Wachstum des Eikernes und des Nukleolus noch etwa parallel laufen. 3: Älteres Stadium. 4: Älteres Stadium aus dieser Gonade.

\section{Diskussion des Ergebnisses}

Das Ergebnis spricht für die Auffassung von Jörgensen: die Masse des Kernes und die Masse des Nukleolenmaterials sind unabhängig von einander.

Ob man diesen Satz durchaus verallgemeinern darf, können nur weitere Untersuchungen an anderen Objekten zeigen. Doch sprechen die rein optisch erlangten Ergebnisse von JörGENSEN an anderen Eiern dafür, daß man diesen Satz wenigstens auf das Ei ausdehnen darf. 
Wohl aber ist eine andere Einschränkung notwendig: die Eier zeigen den bekannten Riesenwuchs, wobei Kerne und Plasma anfänglich in einem festen Größenverhältnisse stehen, dann aber häufig

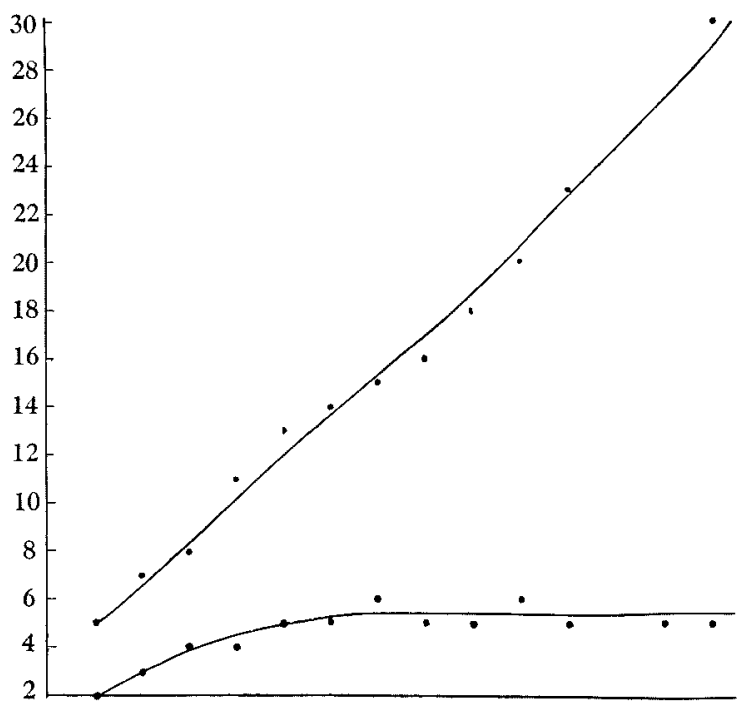

Abb. 5: Kurven des Wachstums von Eikernen ihren dazu gehörigen Nukleoli: Punkte auf der gleichen Abszisse gehören demselben $\mathrm{Ei}$ an, geben also die Relation der Größe desselben Systems wieder. das Plasma den Kern noch überflügelt. Zwar sprechen die Befunde bei anderen Zellen auch dafür, daß keine feste Beziehung zwischen Kerngröße und Nukleolenmasse besteht; aber es sind hier jedenfalls noch genauere Messungen nötig.

Und doch kann man nach dem, was wir bisher wissen, wohl schon sagen, daß nur in seltenen Fällen Kerne und Nukleolen in einem festen Größenverhältnisse zu einander stehen.-

G. HERTwIG kommt in seiner Übersicht über den gegenwärtigen Stand der Nukleolenfrage 1929 zu dem Ergebnis: „Darin stimmen alle nukleolenartigen Gebilde überein, daßs sie kein irgendwie geartetes Kernteilkörpermaterial enthalten; sie sind vielmehr nur aus paraplasmatischem Material aufgebaut". Welches ist nun nach dem Autor das Kennzeichen eines „Teilkörpermaterials“? „Das Protoplasma“, sagt G. HERTWIG, „das Teilkörpermaterial ist, wird repräsentiert durch die Protomeren; das Protoplasma besteht aus Ionen, Atomen, Molekulen und Molekülkomplexen“. G. HERTWIG versteht also unter „Teilkörper" nur die Protomeren HEIDENHaINs (auch wenn er sie im Gegensatz zu HEIDENHAIN als „nichtlebend“ ansieht; das tut nichts zur Sache): d.h. also jene Molekülkomplexe, welche z.B. einen Kern in regelmäßigen Sprüngen von 700, 1400, 2800, 5600 und 11200 aufbauen sollen (JACOBJ, 1926). Geht man von dieser Definition des „Teilkörpers" aus, dann allerdings hat G. HERTWIG recht, wenn er dem Nukleolus keine Eigenschaft als "Teilkörper" zuschreibt, denn die Kurve Abb. 5 zeigt ja, daß keine sprungweise Größenvariation am Nukleolus zu beobachten ist. Gut; wir möchten 
jedoch den Begriff ,P artielles System" anders definieren als „Teilkörper" Für uns ist das Partielle System gegenüber dem umfassenden System gekennzeichnet durch die folgenden Punkte:

1. Es ist ein Teil eines höheren System, welches das partielle System umgreift (Enkapsis);

2. das partielle System steht zum höheren System in einem gewissen Verhältnis der Abhängigkeit und der der Freiheit. Diejenigen Prozesse, in welchen das partielle System frei ist, bestimmen seine Autonomie; in welchem es aber vom höheren System abhängig ist, bestimmen seine Heteronomie.

Auf Grund des obigen Befundes können wir also sagen: hinsichtlich seiner Masse ist das partielle System $\mathrm{Nukleolus} \mathrm{auto-}$ nom gegenüber dem umfassenden System Kern.

MARTIN HEIDENHAIN hat Kerngröße gleich Chromatinmasse gesetzt. Da sich nun herausgestellt hat, daß diese Gleichsetzung nicht besteht, so müssen wir die These von M. HEIDENHAIN einschränken und fragen: steht die Chromatinmasse in einem festen Verhältnisse zur Nukleolenmasse? Diese Frage ist ebenso berechtigt wie die Frage: in welchem Verhältnis steht die Nukleolenmasse zur Masse des Kernsaftes oder des Linins? Diese Fragen haben alle dieses gemeinsam: steht die Größe des Nukleolus in einem festen Verhältnisse zu jenen anderen partiellen Systemen, welche den Kern als Ganzes aufbauen? Diese Frage können wir vorläufig noch nicht beantworten. Wir können hier nur das Eine sagen: das Partialsystem Nukleolus ist in seiner Größe nicht abhängig von dem höheren System Kern. Daß andererseits der Nukleolus in anderer Hinsicht dem Kernsystem gegenüber heteronom ist, das zeigt sich aus seinem Verhalten bei der Mitose und aus manchen anderen Stoffwechselerscheinungen. Darüber zu sprechen ist hier nicht der Ort.

Der Nukleolus ist das Stiefkind der Cytologie. Es ist zweifelhaft, ob die sehr weitgehenden Hypothesen über seine Bedeutung, z. B. aus der Feder von JöRGENSEN oder neuerdings von SAGUCHI, alle richtig sein müssen. Wohl aber ist es notwendig, den Nukleolus intensiver zu untersuchen.

Wir haben uns mit den Nukleolen in den Eiern von Lima auch noch in anderer Hinsicht beschäftigt und werden demnächst unsere Beobachtungen über den Wechsel der Struktur der Nukleolen während des Wachstums vorlegen.

\section{Zusammenfassung}

Die Frage: ist die Größe des Nukleolus abhängig von der Größe des Kernes, erscheint wichtig für die Beurteilung des Verhältnisses 
des umfassenderen Systems Kern zu seinem Partialsystem Nukleolus.

Messungen an den wachsenden Eiern von Lima hians (Lamelli branchiate) zeigten, daß kein festes Größenverhältnis zwischen Kern und Nukleolus besteht: der Eikern wächst gleichmäBig an (Abb. 5) ; der Nukleolus wächst zuerst parallel damit, bekommt aber schnell seine endgültige Größe, auf welcher er stehen bleibt. Die Masse des Nukleolus ist also in diesem Falle unabhängig von der Masse des Kernes.

Es werden die Möglichkeiten der Autonomie und Heteronomie des Nukleolus diskutiert, und der Begriff Partielles System wird definiert.

\section{Zitierte Schriften}

Bauer, H.: Oozytenkerne von Insekten. Z. Zellforschung 18, 1933.

Hartmann, O.: Zelle, Kern-und Nukleolengröße. Arch. f. Zellf. 15, 1921.

Heidenhain, M.: Plasma und Zelle, Jena 1907.

Hertwig, Günther: Die lebende Masse. Hdb. d. mikr. Anat., Berlin 1929.

Jörgensen, M.: Zellenstudien I. Arch. f. Zellforsch. 10, 1913.

Lenoir, M. : Composants dermatiques du noyau. Rev. gén. Bot. 43, 1931.

Saguchi, S.: Biol. Bedeutung des N'ukleolus. Zytol. Studien 7, 1934. 